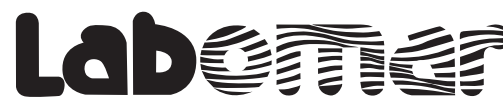

Arquivos de Ciências do Mar

\section{RENDIMENTO CORPORAL DO BAGRE MARINHO, Sciades herzbergii}

\author{
Body yield of sea catfish, Sciades herzbergii \\ Marcony Barreto Vasconcelos-Filho', Caio Rubens do Rêgo Oliveira', \\ Catarina Cardoso de Melo', Marcos Antônio Pergentino da Silva1, \\ Humber Agrelli de Andrade ${ }^{2}$, Paulo Roberto Campagnoli Oliveira Filho ${ }^{3 *}$ \\ ${ }^{1}$ Laboratório de Tecnologia do Pescado, Departamento de Pesca e Aquicultura, Universidade Federal Rural de Pernambuco, Recife \\ Av.Dom Manoel de Medeiros, km 8, Dois Irmãos, PE, 52171-900. E-mail: marconybvf@hotmail.com \\ 2 Professor Adjunto da Universidade Federal Rural de Pernambuco, Campus sede, Av. Dom Manoel de Medeiros, km 8, Dois Irmãos, \\ Recife, PE 52171-900. E-mail: humber.andrade@gmail.com \\ ${ }^{3}$ Professor Adjunto da Universidade Federal Rural de Pernambuco, Campus sede, Av. Dom Manoel de Medeiro, km 8, Dois Irmãos, \\ Recife, PE 52171-900. E-mail: paulocoliveira79@hotmail.com \\ ${ }^{*}$ Autor correspondente.
}

\begin{abstract}
RESUMO
O objetivo do presente estudo foi avaliar o rendimento corporal do bagre marinho, Sciades herzbergii, com o intuito de obter informações capazes de agregar valor a este pescado, utilizando as seguintes estimativas: rendimento de filé, músculo abdominal, pele, cabeça, vísceras e espinhaço. O experimento foi realizado utilizando 48 exemplares de bagres com peso variando entre 79 e 503 g adquiridos de pescadores artesanais no município de Sirinhaém, Pernambuco. Os rendimentos médios foram: vísceras $(12,1 \%)$, músculo abdominal $(7,0 \%)$, filé $(20,1 \%)$, pele $(6,2 \%)$, cabeça $(33,8 \%)$ e espinhaço $(22,8 \%)$. Estes resultados foram semelhantes ao rendimento corporal de outras espécies de peixes de interesse comercial. Portanto, o bagre marinho apresenta um potencial para o aproveitamento tecnológico na forma de filés, produtos de alto valor agregado ou então para o aproveitamento dos resíduos na elaboração de farinha ou óleo de pescado.
\end{abstract}

Palavras-chave: Tecnologia, processamento, aproveitamento integral, filé de pescado.

\begin{abstract}
The aim of this study was to evaluate the body yield of sea catfish, Sciades herzbergii, in order to obtain some information for add value to this fish, using the following estimates: yield of fillet, abdominal muscle, skin, head, gut and backbone. The experiment was conducted using 48 catfish weighing between 79 and $503 \mathrm{~g}$, acquired from artisanal fishermen in the municipality of
\end{abstract}

Recebido: 27 ago 2015

Aceito: 31 jan 2017

Publicado online: 31 mai 2017 
Sirinhaém, Pernambuco. The mean yields were viscera (12.1\%), abdominal muscle $(7.0 \%)$, fillet $(20.1 \%)$, skin $(6.2 \%)$, head (33.8\%) and spine (8\%). These results were similar to the body similar of other commercial fish species. Therefore, sea catfish presents a potential for technological use in the form of steaks, high value-added products and then to the recovery of waste in the preparation of flour or fish oil.

Keywords: Technology, processing, integral use, fish fillet.

\section{INTRODUÇÃO}

O pescado (peixes, crustáceos, moluscos, anfíbios, quelônios e mamíferos de água doce ou salgada usados na alimentação humana) é um alimento que se destaca pela quantidade e qualidade das proteínas, vitaminas e minerais. Além disso, o consumo frequente deste tipo de carne previne doenças cardiovasculares e tem elevada importância nas fases iniciais do desenvolvimento humano (Sartori \& Amancio, 2012).

A Organização Mundial de Saúde (OMS) recomenda que para haver uma boa nutrição, o consumo mínimo de pescado deve ser de $12 \mathrm{~kg} / \mathrm{hab} /$ ano. Observa-se que o consumo médio mundial é de 20,1 kg/hab/ano (FAO, 2016), no entanto no Brasil o consumo de pescado é de aproximadamente $10 \mathrm{~kg} / \mathrm{hab} / \mathrm{ano}(\mathrm{FAO}, 2016)$, ou seja abaixo do mínimo recomendado.

O baixo consumo de pescado em relação a outros alimentos de origem animal no Brasil está associado ao preço elevado, falta de hábito, pouca oferta de produtos de boa qualidade e baixa diversidade e praticidade dos produtos oferecidos (Honorato et al., 2014).

A fim de minimizar este problema, estudos que avaliem aspectos de rendimento corporal de espécies de pescado são de grande importância, pois podem fornecer dados sobre as partes comestíveis, assim como informações para o aproveitamento integral dos resíduos. Com isso, pode-se sugerir, por exemplo, se o processamento adequado de determinada espécie se dá na forma de filés, postas, produtos derivados ou mesmo, se a mesma é indicada para a elaboração de farinha de peixe.

Nesse contexto, pode-se ressaltar a espécie Sciades Herzbergii, conhecido como bagre marinho. Este peixe pertence à ordem dos Siluriformes, família Ariidae e apresenta distribuição ao longo de toda costa Atlântica. Habita a zona litorânea pouco profunda com fundos lodosos ou arenosos e em geral procura a foz de rios e regiões lagunares para fazer a desova. Como principais características morfológicas, o bagre marinho, S. herzbergii, apresenta um par de barbilhões na maxila superior e um ou dois pares na maxila inferior e nadadeiras peitoral e dorsal com a presença de espinho (Nelson, 1994).

Os bagres ocupam a 11. a posição entre as espécies de peixes marinhos mais capturados, com produção de 10.108 toneladas ou seja, 1,97\% do total da pesca marinha brasileira (MPA, 2011). A maioria destes peixes é oriunda da pesca artesanal, em que a espécie é considerada fauna acompanhante (by-catch) nas operações direcionadas para a captura de camarões. Quando parte da fauna acompanhante é devolvida ao mar, a mesma é denominada de rejeito ou descarte. O rejeito ocorre por falta de interesse econômico e/ou tecnológico. A prática é frequente na pesca do camarão no município de Sirinhaém, litoral sul de Pernambuco, onde grande parte dos bagres pescados são descartados.

Segundo o Código de Conduta para a Pesca Responsável, uma das maiores preocupações está no desperdício de proteína constituinte da fauna acompanhante. Essa preocu- 
pação está ligada principalmente aos impactos ambientais da atividade pesqueira e ao desperdício do pescado, que cresce em importância frente ao fato de que existem cerca de 805 milhões de pessoas no mundo que sofrem de desnutrição crônica (FAO, 2015).

A aparência pouco atrativa e a presença de espinhos nas nadadeiras peitorais e dorsal são fatores que influenciam na baixa aceitação dos bagres. De fato, não foram encontrados na literatura estudos sobre o aproveitamento tecnológico dos bagres marinhos. Portanto, o objetivo do presente estudo foi avaliar o rendimento corporal do bagre marinho, Sciades herzbergii, a fim de se levantar dados relevantes para agregar valor na forma de filés, produtos elaborados ou então para o aproveitamento dos resíduos.

\section{MATERIAL E MÉTODOS}

Para o experimento foram utilizados 48 exemplares de bagres marinhos, Sciades herzbergii, pesando entre 79 a 503 g, adquiridos com o máximo de frescor de pescadores artesanais do município de Sirinhaém, litoral sul de Pernambuco, no período de março a abril de 2013. Os peixes foram acondicionados em caixa térmica com gelo em escamas na proporção de 1:1 (relação de gelo:peixe) e levados até o Laboratório de Tecnologia do Pescado, pertencente ao Departamento de Pesca e Aquicultura da Universidade Federal Rural de Pernambuco, Recife. Em laboratório, foi realizada a lavagem dos peixes com água clorada (5 ppm) para a retirada do muco superficial e demais impurezas. Após a limpeza foi realizado o processamento dos bagres sendo cada etapa realizada sempre pelo mesmo pesquisador, com a finalidade de evitar variações decorrentes do envolvimento de diferentes manipuladores. A sequência do processo de filetagem dos bagres marinhos é mostrada no

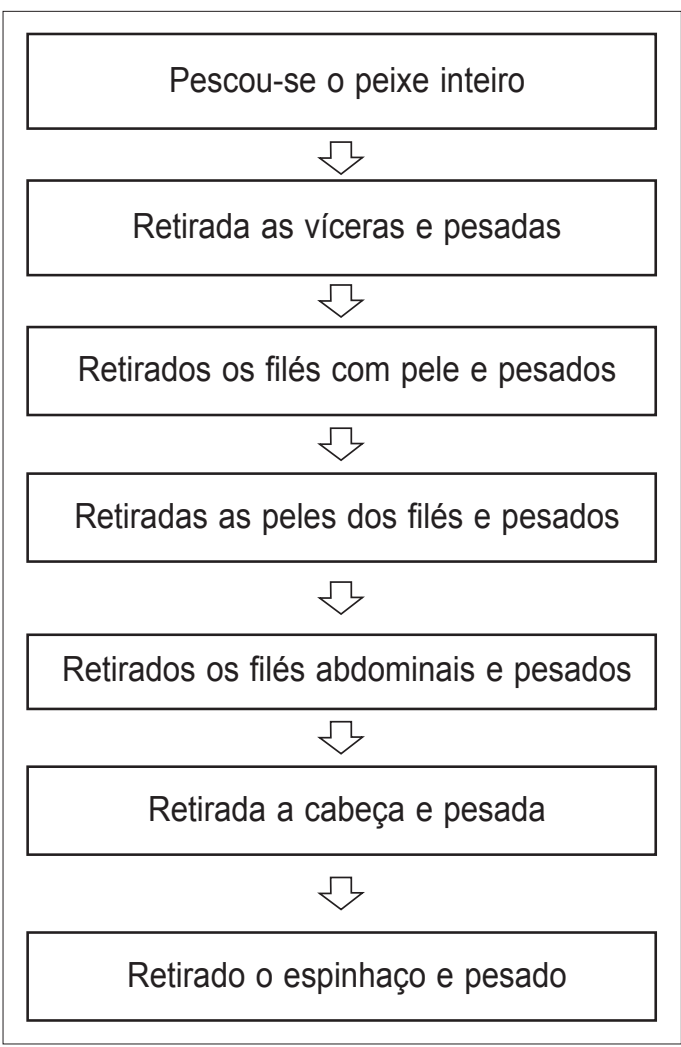

Figura1-Fluxograma das etapas de processo defiletagem dos bagres marinhos, Sciades herzbergii. fluxograma da Figura 1 e ilustrada na Figura 2.

Os exemplares de bagres marinhos e as partes processadas foram pesados em uma balança com precisão de 0,01g (Shimadzu $\left.{ }^{\circledR}\right)$. Posteriormente foram calculadas as proporções entre os pesos de cada uma das partes corporais estudadas em relação ao peso total de acordo com as seguintes equações: Rendimento de filé $=$ (peso do filé $/$ peso do peixe inteiro) $x$ 100; Rendimento de músculo abdominal = (peso do músculo abdominal/peso do peixe inteiro) $\times 100 ;$ Rendimento de pele $=($ peso da pele/peso do peixe inteiro) $x$ 100; Rendimento de cabeça $=$ (peso da cabeça $/$ peso do peixe inteiro) $\times 100$; Rendimento de vísceras $=$ (peso das vísceras/peso do peixe inteiro) x 100 e Rendimento de espinhaço $=$ (peso do espinhaço/peso do peixe inteiro) $\times 100$.

As relações entre o peso total e os rendimentos das diferentes partes do corpo foram avaliadas a partir do ajuste e seleção de modelos. Para isso foram considerados os modelos linear $(\mathrm{y}=a+b x)$, exponencial $\left(y=a e^{b x}\right)$ e geométrico $\left(y=a x^{b}\right)$, e o melhor deles foi selecionado com base no cálculo do coeficiente de 
determinação $\left(\mathrm{R}^{2}\right)$. Para todos os modelos foi realizado o teste $\mathrm{F}$ para verificar se as regressões eram significativas com nível de significância de $\alpha=0,05$. No caso da rejeição da hipótese nula isto significaria que há forte associação entre as variações de peso e dos rendimentos das diferentes partes do corpo. Em caso contrário, isto significaria que o rendimento (proporção da parte do corpo em relação ao peso total), é similar para exemplares de diferentes pesos e tamanhos. Todas as análises foram realizadas com o programa estatístico $\mathrm{R}$ (R Core Team, 2015).

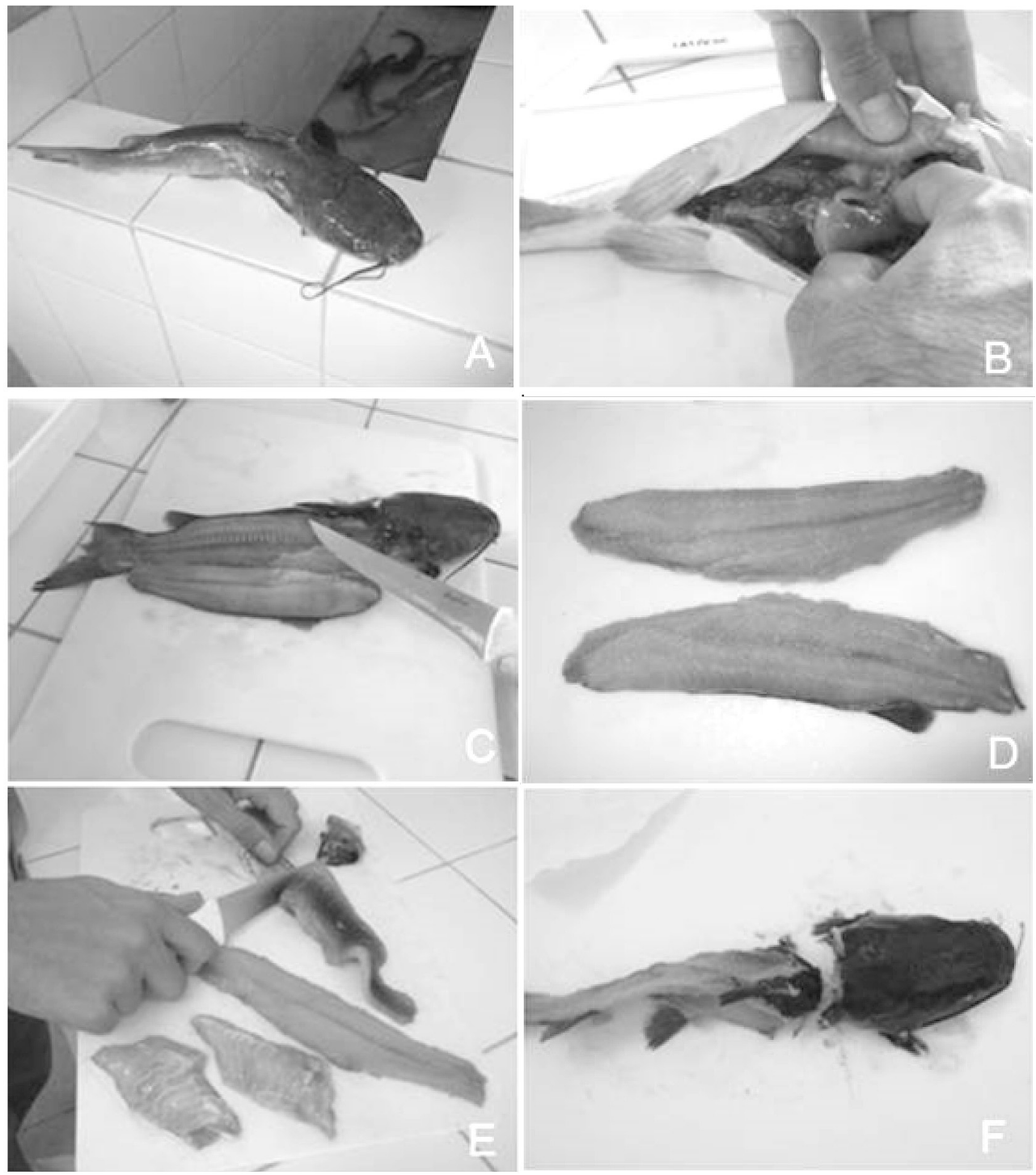

Figura 2 - Etapas do processamento dos bagres marinhos, Sciades herzbergii: A - detalhe do exemplar da espécie, $\mathbf{B}$ - retirada das vísceras, $\mathbf{C}$ - retirada dos filés, $\mathbf{D}$ - detalhe dos filés com pele, E - retirada da pele e detalhe do músculo abdominal, $\mathbf{F}$ - retirada da cabeça.

\section{RESULTADOS E DISCUSSÃO}

Na análise da relação entre o peso total e os rendimentos das diferentes partes do corpo (Tabela I), dentre os três modelos considerados, o geométrico foi o de melhor desempenho (maior $\mathrm{R}^{2}$ ) entre o peso total e os rendimentos das vísceras e do espinhaço e o mo- 
delo linear entre o peso total e o rendimento da cabeça. Entretanto, o aumento do peso total dos bagres não está relacionado a variações sistemáticas dos valores de rendimento de filé $(20,1 \%)$, músculo abdominal $(7,0 \%)$ e pele $(6,2 \%)$, ou seja, não há associação entre peso total e estas três variáveis.

Tabela I - Relações entre o peso total e os rendimentos de filé, músculo abdominal, pele, cabeça, vísceras e espinhaço do bagre marinho, Sciades herzbergii.

\begin{tabular}{|c|c|c|c|c|}
\hline & Relação & Equação & $\mathrm{R}^{2}$ & p-valor \\
\hline \multirow{18}{*}{$\begin{array}{l}\text { की } \\
\widetilde{\pi} \\
0 \\
0 \\
0 \\
0 \\
0\end{array}$} & \multirow{3}{*}{ Filé (\%) } & $\mathrm{RF}=19,474+0,003 \mathrm{PT}$ & 0,012 & 0,474 \\
\hline & & $\mathrm{RF}=17,747 \mathrm{PT}^{0,022}$ & 0,005 & 0,646 \\
\hline & & $R F=19,206 \exp \left(1,70 \times 10^{-4} \mathrm{PT}\right)$ & 0,015 & 0,434 \\
\hline & \multirow{3}{*}{$\begin{array}{l}\text { Músculo } \\
\text { Abdominal (\%) }\end{array}$} & $\mathrm{RM}=6,803+0,001 \mathrm{PT}$ & 0,008 & 0,560 \\
\hline & & $\mathrm{RM}=4,697 \mathrm{PT}^{0,074}$ & 0,038 & 0,204 \\
\hline & & $\mathrm{RM}=6,602 \exp \left(2,24 \times 10^{-4} \mathrm{PT}\right)$ & 0,017 & 0,397 \\
\hline & \multirow{3}{*}{ Pele (\%) } & $\mathrm{RP}=7,125-0,004 \mathrm{PT}$ & 0,030 & 0,263 \\
\hline & & $\mathrm{RP}=14,677 \mathrm{PT}^{-0,173}$ & 0,055 & 0,124 \\
\hline & & $\mathrm{RP}=6,725 \exp \left(-6,13 \times 10^{-4} \mathrm{PT}\right)$ & 0,034 & 0,234 \\
\hline & \multirow{3}{*}{ Cabeça (\%) } & $\mathrm{RC}=35,600-0,008 \mathrm{PT}$ & 0,100 & 0,036 \\
\hline & & $\mathrm{RC}=41,163 \mathrm{PT}^{-0,038}$ & 0,040 & 0,148 \\
\hline & & $\mathrm{RC}=35,532 \exp \left(2,4 \times 10^{-4} \mathrm{PT}\right)$ & 0,099 & 0,037 \\
\hline & \multirow{3}{*}{ Vísceras (\%) } & $\mathrm{RV}=9,465+0,012 \mathrm{PT}$ & 0,135 & 0,014 \\
\hline & & $\mathrm{RV}=3,154 \mathrm{PT}^{0,248}$ & 0,212 & 0,002 \\
\hline & & $\mathrm{RV}=9,421 \exp \left(9,93 \times 10^{-4} \mathrm{PT}\right)$ & 0,164 & 0,006 \\
\hline & \multirow{3}{*}{$\begin{array}{l}\text { Espinhaço } \\
(\%)\end{array}$} & $\mathrm{RE}=41,301-0,084 \mathrm{PT}$ & 0,329 & $4,65 \times 10^{-5}$ \\
\hline & & $\mathrm{RE}=1.920,688 \mathrm{PT}^{-0,872}$ & 0,435 & $1,12 \times 10^{-6}$ \\
\hline & & $\mathrm{RE}=42,913 \exp \left(-3,69 \times 10^{-3} \mathrm{PT}\right)$ & 0,376 & $9,49 \times 10^{-6}$ \\
\hline
\end{tabular}

\section{Rendimento de filé e músculo abdominal}

O rendimento de filé obtido para o bagre no presente estudo é semelhante ao encontrado para outros peixes teleósteos comerciais. O rendimento de filé da tilápia do Nilo varia de 30 a 35\% (Viegas et al., 2013), o jaraqui, Semaprochilodus insignis, entre 34,9 a 41,8\% (Costa et al., 2014), o pacu, Piaractus mesopotamicus, apresenta média de 25,2\% (Lima et al., 2012) e o tambaqui, Colossoma macropomum, foi estimado em 27,8\% (Mujica \& Lima, 2011). No entanto, em outras espécies de bagres os resultados mostram grande variação. Por exemplo, para o surubim pintado, Pseudoplatystoma corruscans, o rendimento de filé é de 33,2\% (Frascá-Scorvo et al., 2008), para o surubim cachara, Pseudoplatystoma fasciatum, 47,8\% (Burkert et al., 2008), para o barbado, Pinirampus pirinampu, entre 38,3 a 42,2\% (Adames et al., 2014) e para o bagre africano, Ictalurus punctatus, 39,6\% (Souza et al., 1999). Esta variação no rendimento dos filés dos bagres encontrados nos diversos estudos pode refletir os diferentes métodos utilizados, e se considera a soma dos dois tipos de filés (lateral e abdominal) ou o peso separado. O músculo abdominal (filé localizado na parte ventral do peixe) é uma característica morfológica de algumas espécies de peixes, dentre eles os bagres. É um tipo de corte que vem sendo apreciado pelo consumidor pelo sabor diferenciado (Fantini et al., 2014).

Apesar da aparência externa pouco atrativa do bagre, S. herzbergii, os filés apresentaram características sensoriais interessantes, tais como: coloração clara levemente rosada, textura firme e ausência de espinhos intramusculares. Portanto, há fatores favoráveis para elaboração de produtos de valor agregado como embutidos ou empanados. Além disso, o processo de filetagem é de fácil execução, pois não há escamas e a consistência da carne é firme. 


\section{Rendimento da pele}

O rendimento médio da pele dos bagres marinhos foi de 6,2\%. Este valor é próximo ao encontrado por Fantini et al. (2014) e Burkert et al. (2008) em estudo de rendimento do surubim, com rendimento de pele de 6,7 e 7,1\%, respectivamente. Para a tilápia do Nilo, Oreochromis niloticus, observou-se resultados de rendimento de pele entre 4,8 a 5,7\% (Viegas et al., 2013). Portanto, os valores de rendimento de pele de bagres foram próximos aos resultados encontrados para outros peixes teleósteos. O conhecimento do rendimento em pele é importante, pois algumas espécies são comercializadas sob a forma de filé com pele. Outro interesse crescente está em aproveitar a pele para ser utilizada como matéria-prima para a obtenção de colágeno (Souza \& Inhamuns, 2011; Fantini, 2014). Além disso, a pele dos peixes é um produto de alta qualidade, resistência e de característica peculiar, podendo ser utilizada para o curtimento em couro que é empregado na elaboração de vários produtos tais como: bolsas, sapatos, jaquetas e vestidos.

\section{Rendimento da cabeça}

A cabeça é um dos fatores determinantes na seleção de uma espécie para processamento e comercialização, pois é inversamente proporcional ao rendimento de filé. O rendimento de cabeça do $S$. herzbergii foi em média de 33,8\%, e não apresentou grandes variações em função do tamanho dos exemplares. Em estudo com o bagre africano, Clarias gariepinus, observou-se rendimento de cabeça de 34,5\% para peixes com 800 gramas (Souza et al., 1999), ou seja, próximo dos valores obtidos no presente estudo. No entanto, para o barbado, Pinirampus pirinampu, o rendimento de cabeça varia entre 11,3 a 12,1\% (Adames et al., 2014), o pacu, Piaractus mesopotamicus, é de 20,8\% (Lima et al., 2012), para o tambaqui, Colossoma macropomum, é de 24,4\% (Mujica \& Lima 2011), para a tilápia, Orechromis niloticus, em torno de 18,1\% (Viegas et al., 2013) e para a viola, Loricariichthys anus, é entre 18,4 a 19,4\% (Britto et al., 2014). Isto mostra que a conformação da cabeça das diferentes espécies pode causar diferenças nos rendimentos deste quesito corporal e que os bagres marinhos da espécie $S$. herzbergii apresentam rendimento de cabeça superior aos demais teleósteos.

\section{Rendimento das vísceras}

A associação entre o peso dos exemplares e o rendimento das vísceras é positiva, o que implica que a participação percentual da víscera no peso total aumenta à medida que o bagre é mais pesado. No entanto, a relação não é linear e o aumento da participação percentual das vísceras em função do peso é menos acelerado em exemplares de maior porte. Basso et al. (2011) também verificaram aumento da porcentagem de vísceras conforme houve aumento do peso do pacu, Piaractus mesopotamicos. Os autores sugerem que esse aumento no rendimento de vísceras é decorrente do estágio de maturação das gônadas. Concordando com esta afirmação, no presente estudo, foi possível constatar a presença de bagres marinhos com gônadas em estágio de maturação avançado, o que interferiu no rendimento de vísceras. Para outras espécies de peixes, o rendimento de vísceras é próximo aos resultados alcançados no presente estudo. Mujica \& Lima (2011) encontraram rendimento de 12,1\% de vísceras para o tambaqui, Colossoma macropomum e Freato et al. (2005) com valores entre $10,1 \%$ e $12,2 \%$ para a piracanjuba, Brycon orbignyanus. Por outro lado, Souza et al. (1999) observaram rendimento de 7,7 a 8,9\% das vísceras do bagre africano, Clarias gariepinus, assim como Adames et al. (2014) para o armado, Pinirampus pirinampu, que é de 8,6 a $10,0 \%$. 


\section{Rendimento do espinhaço}

A participação relativa percentual do espinhaço diminui em função do peso do total, o que caracteriza uma associação negativa entre as variáveis. No entanto, cabe mencionar, que a relação não é linear e o decaimento da participação percentual do espinhaço no peso total é menos acelerado quando se trata de exemplares de maior porte. Como as associações entre peso e as demais variáveis (i.e. filé) não foram significativas, é aparente que o decréscimo da participação do espinhaço é um contrapeso ao aumento da participação relativa das vísceras. Assim, o decréscimo da importância relativa do espinhaço para animais de maior porte não se contrapõe a um maior aproveitamento de material mais nobre (i.e. filé), e sim de vísceras.

Em pintados, Pseudoplatystoma corruscans, o rendimento de espinhaço é de aproximadamente 44,8\% (Frascá-Scorvo et al., 2008). Comparativamente, este valor é próximo dos menores exemplares de bagres do presente estudo. No entanto, para a tilápia do Nilo, Oreochromis niloticus, o rendimento de espinhaço é de aproximadamente 21\% (Viegas et al., 2013). Portanto, as informações disponíveis indicam que o rendimento de espinhaço está associado com a espécie e o peso dos peixes.

Tecnologias têm surgido para a utilização mais eficiente dos resíduos advindos do beneficiamento do pescado como fontes alimentares, transformando-os em produtos nutritivos e com boa aceitação de mercado. O espinhaço com carne aderida após a retirada do filé tem sido cada vez mais utilizado para obtenção da carne mecanicamente separada (CMS), que é a matéria-prima inicial utilizada na elaboração de produtos de valor agregado como empanados, embutidos e reestruturados (Kirschnik et al., 2013).

\section{CONCLUSÃO}

O bagre marinho, Sciades herzbergii, apresenta rendimento corporal semelhante ao de outras espécies de peixes teleósteos de interesse comercial, sendo, portanto, uma espécie com potencial para o aproveitamento tecnológico na forma de filés, produtos de alto valor agregado ou então para o aproveitamento dos resíduos na elaboração de farinha ou óleo de pescado.

\section{REFERÊNCIAS BIBLIOGRÁFICAS}

Adames, M.S.; Krause, R.A.; Damasceno, D.Z.; Piana, P.A.; Oliveira, J.D.S. \& Bombardelli, R.A. Características morfométricas, rendimentos no processamento e composição centesimal da carne do barbado. B. Inst. Pesca, São Paulo, v.40, n.2, p.251 - 260, 2014.

Basso L.; Ferreira M.W. \& Silva, A.R. Efeito do peso ao abate nos rendimentos dos processamentos do Pacu (Piaractus mesopotamicos). Arq. Bras. Med. Vet. Zoo. Belo Horizonte, v.63, n.5, p.1260-1262, 2011.

Britto, A.C.P.; Rocha, C.B.; Tavares, R.A.; Fernandes, J.M.; Piedras, S.R.N. \& Fernandes, J.L.O. Rendimento corporal e composição química do filé da viola (Loricariichthys anus). Cienc. Anim. Bras., Goiânia, v.15, n.1, p. 38-44, 2014.

Burkert, D.; Andrade, D.R.; Sirol, R.N.; Salaro, A.L.; Rasguido, J.E.A. \& Quirino, C.R. Rendimentos do processamento e composição química de filés de surubim cultivado em tanques-rede. Rev. Bras. Zootecn., Viçosa, v.37, n.7, p.1137- 1143, 2008. 
Costa, T.V.; Machado, N.J.B.; Brasil, R.J.M. \& Fragata, N.P. Caracterização físico-química e rendimento do filé e resíduos de diferentes espécies de jaraqui (Semaprochilodus spp.). B. Inst. Pesca, São Paulo, v.40, n.1, p.35 - 47, 2014.

Fantini, L.E.; Oliveira, C.A.L.; Rodrigues, R.A.; Zardin, A.M.S.O.; Ushizima, T.T. \& Campos, C.M. Rendimento de carcaça de surubins Pseudoplatystoma spp. produzidos em viveiros sob diferentes densidades de estocagem. Semina, Londrina, v. 35, n. 5, p. 2769-2780, 2014.

FAO, 2015. The State of Food Insecurity in the World. Meeting the 2015 international hunger targets: taking stock of uneven progress. Roma. 62 págs.

FAO. 2016. The State of World Fisheries and Aquaculture 2016. Roma. 200 págs.

Frascá-Scorvo, C.M.D.; Baccarin, A.E.; Vidotti, R.M.; Romagosa, E.; Scorvo-filho, J.D. \& Ayroza, L.M.S. Influência da densidade de estocagem e dos sistemas de criação intensivo e semi intensivo no rendimento de carcaça, na qualidade nutricional do filé e nas características organolépticas do pintado Pseudoplatystoma corruscans. B. Inst. Pesca, São Paulo, v.34 n.4, p. 511 - 518, 2008.

Freato, T.A.; Freitas, R.T.F.; Santos, V.B.; Logato, P.V.R. \& Viveiros, A.T.M. Efeito do peso de abate nos rendimentos do processamento da piracanjuba (Brycon orbignyanus, Valenciennes, 1849). Cien. Agrotec., Lavras, v. 29, n. 3, p. 676-682, 2005.

Honorato, C.A.; Smerman, W.; Angélici, A.F. \& Dal Bem, C.R. Efeito das classes de peso sobre o rendimento de processamento de tucunaré (Cichla sp.). Sci. Agrar. Paran. Marechal Candido Rondon, v.13, n.1, p. 65-70, 2014.

Kirschnik, P.G.; Trindade, M.A.; Gomide, C.A.; Moro, M.E.G. \& Viegas, E.M.M. Estabilidade em armazenamento da carne de tilápia-do-nilo mecanicamente separada, lavada, adicionada de conservantes e congelada. Pesq. Agropec. Bras., Brasília, v.48, n.8, p.935-942, 2013.

Lima, M.M.; Mujica, P.I.C. \& Lima, A.M.; Caracterização química e avaliação do rendimento em filés de caranha (Piaractus mesopotamicus). Braz. J. Food Technol., Campinas, IV SSA, p. 41-46, 2012.

Ministério da Pesca e Aqüicultura - MPA. Boletim Estatístico da Pesca e Aquicultura. Brasília: MPA, 2011. Disponível em: http://www.icmbio.gov.br/cepsul/images/stories/biblioteca/download/estatistica/est_2011_bol__bra.pdf. Acesso em: 15/04/2016.

Mujica, P.I.C. \& Lima, M.M. Caracterização físico-química e avaliação do rendimento em filés de tambaqui (Colossoma macropomum). In Anais do Congresso Sergipano de Ciências, Aracajú, 2011.

Nelson, J.S. 1994. Fishes of the World. Third edition. John Wiley \& Sons, Inc., New York. 600 p.

R Core Team; R: A Language and Environment for Statistical Computing. Viena, Austria. http://www.R-project.org/. 2015.

Sartori, A.G.O \& Amancio, R.D., Pescado: importância nutricional e consumo no Brasil. Rev. Seg. Alim. Nutri., Campinas, v.19. n. 2 p. 83-93, 2012.

Souza, A.F.L. \& Inhamuns, A.J. Análise de rendimento cárneo das principais espécies de peixes comercializadas no Estado do Amazonas, Brasil. Acta Amaz. Manaus, v. 41, p. 289 296, 2011.

Souza, M.L.R.; Lima, S.; Furuya, W.M.; Pinto, A.A.; Loures, B.T.R.R. \& Povh, J. A. Estudo 
de carcaça do bagre africano (Clarias gariepinus) em diferentes categorias de peso. Acta Sci., Maringá, v. 21, n. 3, p. 637-644, 1999.

Viegas, E.M.M.; Carvalho, M.R.B.; Oliveira Filho, P.R.C.; Kirschnik, P.G.; Aiura, F.S. \& Vargas, S.C. Changes during chilled storage of whole tilapia and short-term frozen storage of tilapia fillets. J. Aquat. Food Prod. T, Raleigh, v. 22, p. 192-200, 2013. 